\title{
Surface Abundance Anomalies of Main Sequence Stars in Globular Clusters - A Probe into Interactions between Stars
}

\author{
Masaaki Shimada \\ Department of Liberal Arts, Nagoya College, Toyoake, Aichi, Japan \\ Masayuki Y. Fujimoto, Shimako Yamada \\ Department of Physics, Hokkaido University, Sapporo, Hokkaido, Japan \\ Daiichiro Sugimoto \\ University of Air, Mihamaku, Chiba, Japan
}

\begin{abstract}
In most of globular clusters, surface abundance anomalies are observed not only from red giant stars but also from main sequence stars. We discuss the possibility that the latter anomalies can be explained in terms the pollution due to mass transfer during close encounters with red giants, the latter of which have already developed the anomalies through their internal processes. If this is the case, the main sequence stars with the abundance anomalies may serve as a probe into the star-star interactions in dense cores of globular clusters.
\end{abstract}

\section{Introduction}

There have been growing evidence pointing to the importance of star-star interactions in the globular clusters. The blue stragglers on the brighter side of turn-off stars of the main sequence in HR diagram are thought to form from collisional coalescences of stars and/or binary merging (e.g., Bailyn 1995). The numbers of known BSs have recently been raised greatly through the HST observations, up to hundreds ( 300 for M80 Ferraro et al. 1999). Their presence is indicative of much greater aggregations of less massive correspondences along the main sequence below the turn-off, though hardly distinguished from others.

Another evidence is a large star-to-star variation of surface abundances among red giants in some clusters. It includes $\mathrm{Mg}$ and $\mathrm{Al}$, which demand much higher temperatures than realized in red giants within the current standard framework of stellar evolution. Further such anomalies have never been found from field giants (e.g., Da Costa 1997). The model of deep mixing with the interactions with environment stars taken into account is proposed and shown to reproduce the observed anomalies (Fujimoto et al. 1999, Aikawa et al. 2001).

Recently, the same anomalies are reported for turn-off stars and subdwarfs with high dispersion observations using VLT (Gratton et al. 2001). The gravitational potential of these unevolved stars is too shallow to allow such high 
temperatures as necessary to process $\mathrm{Mg}$ and $\mathrm{Al}$. There are no other models that have succeeded in producing these anomalies. It is therefore reasonable to regards the abundance anomalies as being transplanted through mass transfer during a close encounter with the giant that have already developed them. Small mass suffices to disguise their shallow surface convective zone $\left(\sim 10^{-3} M_{\odot}\right)$.

The abundance anomaly among MS stars was already inferred from low dispersion spectra, and in particular, some clusters show nearly equal numbers of CN-weak and CN-strong stars (e.g., 47 Tuc, Briley 1991). This implies very large frequency of star-star interactions in the clusters from our interpretation. In this paper, we evaluate the encounter rates and discuss this possibility.

\section{Rates of Star-Star Interactions}

The collision frequency between MS stars has been discussed by Hills \& Days (1976). We estimate the rates of close encounters between MS and RG stars in the similar way both for the Salpeter and lognormal mass functions.

The results are summarized as follows: (1) If we take the closest approach necessary for the surface pollution as within several RG radii (Davis et al. 1991), then, the numbers of polluted MS stars are larger than those of BSs and their low-mass correspondences formed from the direct collisions: (2) The production rates are much larger for the lognormal mass function than for the Salpeter mass function since the fraction of RG stars is larger: (3) For 10 Gyrs, the polluted MS stars have been accumulated to occupy several percents for an average stellar density of $10^{3} M_{\odot} \mathrm{pc}^{-3}$ and an average velocity of $10 \mathrm{~km} \mathrm{~s}^{-1}$.

\section{Conclusions and Discussion}

An estimate based on simple two-body interactions predicts appreciable portions of MS stars that have experienced close encounters with RG stars and suffered the surface pollution through mass transfer from the latter. These portions will no longer be negligible from the observational viewpoints, but may not large enough to explain their populations observed from some clusters.

There have to be more interactions between stars if our interpretation for the origin of abundance anomalies among unevolved stars is valid. There are several possibilities of enhanced star-star interactions: (1) The actual cross sections for mass transfer from RGs can be larger than assumed here because of the effects of large size of RGs, the resonant encounters with binaries, the multiple encounters, etc.: (2) The clusters may have undergone the stages when star-star interactions were more frequent than inferred form their current status. In fact, the degrees of abundance anomalies and the populations of BSs vary from cluster to cluster without an apparent correlation to their present properties. This is reminiscent of the long-standing second parameter problem on the morphology of horizontal branch stars. These may have common origins. More theoretical and observational efforts are necessary to elucidate these phenomena and to establish the proper understanding of the evolution of stars in the dense environments and of the evolution of clusters as stellar systems. The abundance anomalies among the unevolved stars are expected to play a key role in these investigations since they retain the prints of past interactions. 